\title{
Insulin resistance syndrome in children and adolescents: clinical meaning and indication for action
}

\author{
International Journal of Obesity (2004) 28, 829-832. doi:10.1038/sj.ijo.0802723
}

The insulin resistance syndrome (IRS) or metabolic syndrome in adults is variably defined. ${ }^{1}$ The WHO includes diabetes/impaired glucose tolerance or insulin resistance and two of the following: dyslipidaemia, hypertension, obesity and microalbuminuria. The European Group for the Study of Insulin Resistance (EGIR) includes insulin resistance or hyperinsulinaemia plus two of the following: elevated fasting glucose, dyslipidaemia, hypertension and central obesity. The National Cholesterol Education Programme Adult Treatment Panel III (NCEP ATPIII) includes any three of the following: elevated fasting plasma glucose, hypertriglyceridaemia, low HDL cholesterol, hypertension and central obesity. Thus two of the three adult definitions include insulin resistance, two specify central adiposity and all include hypertension and lipid abnormalities. It would be excellent from a research and comparative viewpoint if there could be an international consensus on the definition of the IRS in children and adolescents.

A more simplified definition of the metabolic syndrome in adults has been suggested - namely the 'hypertriglyceridaemic waist $^{2}$ - a definition that has the attraction of simplicity. Currently, there are no internationally accepted cut points for the waist circumference in children and adolescents that define a risk level, as in adults. Higgins et $a l^{3}$ have suggested that children with $\geq 33 \%$ body fat and a waist circumference of $\geq 71 \mathrm{~cm}$ are more likely than not to have an adverse risk factor profile. Waist circumference in the study from Moreno et $\mathrm{al}^{4}$ appeared the best predictor in children of the presence of metabolic syndrome-associated variables. Recent evidence from the United Kingdom suggests that waist circumference is increasing in children at a greater rate than is their BMI. ${ }^{5}$

The IRS is associated with an increase in morbidity and mortality in populations, and has associated with it a large number of metabolic abnormalities and disease states. These include an increase in inflammatory markers, microalbuminuria and hepatic steatosis, as well as early ischaemic heart disease, type II diabetes, obstructive sleep apnoea and polycystic ovaries. Adolescent offspring of adults with IRS have been shown to have greater adiposity and to be more insulin resistant than offspring of non-IRS adults, but are similar to non-IRS offspring with regard to lipids and blood pressure, a situation that would fit with the concept of development over time. ${ }^{6}$ In children and adolescents, even with overall reduced levels of abdominal fat compared to adults, abdominal fat is positively correlated with an adverse lipid profile and hyperinsulinaemia. ${ }^{7}$

It is reasonable to conjecture that the IRS in children and adolescents is likely to have similar aetiology(ies) to adults and is the same condition but viewed at different time points. The clinical conditions associated with the IRS are not exclusive to adulthood, with a few exceptions such as early ischaemic heart disease and stroke. These latter conditions reflect the time taken to develop the condition, although with evidence that the origins of vascular disease are laid down in childhood. ${ }^{8}$ If the IRS is a continuum throughout life, it is important to understand the keys to its development in childhood, whether these are in any way different to adulthood and how the normal processes of growth and maturation may affect IRS in childhood and adolescence.

In this paediatric focus issue, Lambert et $a l^{9}$ present their findings on the IRS in a sample of children and adolescents from Quebec. What does this study add to our understanding of insulin resistance in childhood, how might this information be used to inform further studies and how might it relate to clinical protocols and practice?

This study provides prevalence data in three age groups, with the emphasis on IRS (not insulin levels alone), is broadly sampled and with good numbers. Two definitions of IRS are considered: (1) the presence of any three or more of hyperinsulinaemia, overweight, high systolic blood pressure, low triglycerides, low HDL-C and impaired fasting glucose; and (2) the presence of hyperinsulinaemia and at least two of the other five risk factors. Thus one definition is potentially exclusive of insulin resistance as defined in the first instance by high insulin levels. The first definition is a less stringent one, and produced an overall prevalence of $14.0 \%$, compared with the second definition of $11.5 \%$. Known diabetes was an exclusion for the study, but the authors do not state if any children with diabetes or with impaired fasting glucose were identified at the time of the study. Fasting insulin and HOMA-IR were both used as surrogate measures of insulin resistance and similar results were obtained in analysis whichever one was used. This finding emphasises the clinical 
utility of a fasting insulin alone compared to a mathematical derivation of fasting glucose and insulin in what is a presumptive non-diabetic population. The work necessary to collect so many blood samples in a paediatric population should never be underestimated and the metabolic variables are a strong feature of this study.

How does HOMA-IR measure up against other measures of insulin resistance in a paediatric population? Using the gold standard for the measurement of insulin resistance, the glucose clamp technique, Moran et al ${ }^{10}$ found that fasting insulin was significantly associated with $\mathrm{M}(R=0.48)$ in $10-$ $14 \mathrm{y}$ olds. This study also found that fasting insulin did not differ between the Tanner stages, in other words the physiological insulin resistance of puberty could not be detected on fasting insulin levels alone but was evident during clamp studies. It is possible that $2 \mathrm{~h}$ post glucose HOMA improves the definition of insulin resistance but would not be practicable in a study such as this. ${ }^{11}$ Log HOMA may be a better estimate of insulin resistance given that the relationship between HOMA and clamp measures of insulin sensitivity is not linear. Sunehag et al ${ }^{12}$ demonstrated some variability in repeated measures in children of both fasting insulin and the insulin clamp technique, but with correlation coefficients between 0.72 and 0.996 , confirming the utility of a single test in time. Overall fasting HOMA-IR is a reasonable measure of insulin resistance and if anything the prevalence of insulin resistance is likely to have been underestimated in this cohort.

There was no significant difference across ages and between sexes for the IRS in this study. This is in apparent contrast to other studies, which have consistently identified a female preponderance in insulin resistance. ${ }^{13,14}$ However, these studies considered insulin resistance per se, whereas Lambert's study focused on a syndrome definition, rather than absolute values for insulin or HOMA-IR. This observation is also the likely reason for the absence of age difference in IRS. The older age groups in Lambert's study do not exhibit a higher prevalence of IRS. Increased insulin resistance is well described in puberty and a gender difference in insulin resistance has been described as persisting in puberty. ${ }^{10}$ A gender difference is also apparent in the prevalence of type II diabetes in children and adolescents, with the condition more prevalent in females. $^{15,16}$

Whether obesity alone explains all the metabolic abnormalities that characterise the metabolic syndrome is a consideration in the pathophysiology of IRS in children and adolescents. Lambert et al's data do not support obesity as defined by BMI as the sole determinant but do suggest that obesity has a stronger contribution to the clustering of risk factors than insulin or HOMA-IR in these age groups. ${ }^{9}$ IRS is a phenotype and must be influenced by the genotype of which there is no singular variety in the vast majority of human obesity. The environment or gene environment interaction must also play a significant role in the expression of IRS. This study adds to the knowledge of IRS by the confirmation that heterogeneity of the syndrome is present from childhood. What role this heterogeneity plays in the expression of future morbidity is currently an interesting but unanswered question.

Factor analysis aims to discover patterns in relationships among variables and to determine if observed variables can be described largely or entirely in terms of a smaller number of variables (factors). Three factors emerged in this study, which also support the concept of IRS as a heterogeneous syndrome. These factors explained about $65 \%$ of the variance and were $\mathrm{BMI} /$ insulin/lipids, $\mathrm{BMI} /$ insulin/glucose and diastolic/systolic blood pressure. According to factor analysis theory, the overlap between two domains (BMI/ insulin) suggests common aetiologies. The finding of multiple factors is not new in the adult literature and supports one previous study that included children and adolescents. ${ }^{17}$

Lambert's study extends the data from the Bogalusa Heart Study (BHS) and others, which describe the clustering and tracking of cardiovascular risk factors in childhood, adolescence and young adulthood. ${ }^{17-20}$ These were relationship and risk focused studies, and not prevalence studies. Age ranges, and not a single age category, were generally used. Fasting insulin remained associated with VLDL independent of age, gender, glucose levels, obesity (and cigarette and alcohol intake) and rose with age. There were racial differences in fasting insulin and the most adverse profiles occurred in white males. The BHS identified HOMA-IR as higher in females, a strong correlation between BMI and insulin resistance, and that clustering of cardiovascular risk factors was least strong around puberty. Although not strictly a longitudinal study, BHS was able to select out subjects who had had both childhood and adult assessment. Children in the top quartile for BMI and insulin were 11.7 and 3.6 times as likely to develop clustering of risk factors as adults, with BMI and insulin the best predictive model. Adjustment for BMI eliminated the influence of childhood insulin on clustering but not vice versa. Additionally, in adults insulin is an independent risk factor for ischaemic heart disease. ${ }^{21}$ While a different analytical methodology was used in the BHS, these findings are congruent with the data by Lambert et al. ${ }^{9}$ Lambert et al provide prevalence data and support for heterogeneity in IRS.

Of practical significance in this study is that skin folds, as a measure of fat distribution, do not have additional and/or greater predictive value in IRS in children and adolescents. On the other hand, the waist circumference is an indirect measure of central adiposity and is correlated with cardiovascular risk in children and adolescents as well as adults. ${ }^{7}$ The correlation between waist and DEXA trunk fat in children and adolescents is $>0.8$ for both genders. ${ }^{22}$ Given that the measure of waist circumference has good reliability and low measurement error, it would be excellent to see this measure used consistently in studies of this nature. As noted previously, there are as yet no accepted cut points for either the classification of obesity or the presence of increased metabolic risk in children, as exist for adults. 
The authors do not provide a specific reason for selection of the three age groups-9,13 and $16 \mathrm{y}$. The age range encompasses the time past normal adiposity rebound, the time where most children will have both entered and completed physical puberty and is within the age range where tracking of weight is well established. IRS is consistently present over the age range and found in at least 1 in every 10 children and adolescents. Adiposity measures increase significantly across insulin quartiles with absolute numbers reflecting the well-known observation that hyperinsulinaemia commonly occurs in obese children and adolescents. This relationship has been described as nonlinear, with modest increases in insulin levels as BMI increases up to the 90th centile, but with a much more pronounced hyperinsulinaemia risk above the 95th and especially the 97 th centile of BMI. ${ }^{23}$

The authors assert in their discussion that 'trends in childhood IRS should be monitored because of their public health and clinical importance', and that 'increased adiposity is strongly associated with clustering of risk factors and concerted efforts by researchers, clinicians, public health and public policy experts are needed to prevent the adverse consequences of obesity on the health and well-being of future generations'.

The prevalence of obesity among children and adolescents is increasing in both developed and developing countries, although both the speed of the increase and the degree of prevalence vary. In the United States, Europe and Australia, the prevalence of overweight has more than doubled over the last decade, with an increase in the percentage of those overweight subjects with obesity. ${ }^{24,25}$ As the prevalence of overweight and obesity increases it can be assumed that so will the prevalence of insulin resistance. Insulin resistance is predictive of type II diabetes ${ }^{26}$ and hyperinsulinaemia in childhood has been found in several populations known to be at high risk for type II diabetes. ${ }^{27}$ The increasing identification of type II diabetes in youth is a concerning consequence of the obesity epidemic in children. The onset of diabetes at a young age increases the risk in early adulthood of vascular disease, and renal and visual impairments. The numbers of pregnancies complicated by diabetes will continue to increase with consequences for both the mother and the offspring. Although factors such as family history and ethnicity play a role, the most important risk factor for type II diabetes in children is obesity, and nearly one-quarter of obese children and adolescents already have impaired glucose tolerance. ${ }^{28}$ However, the overall numbers of type II diabetics among children and adolescents reflect the tip of the iceberg. Many, many more will suffer from insulin resistance and the IRS.

A universally accepted definition for IRS in children and adolescent is urgently required. Such a definition strengthens the epidemiological studies, which will provide information on the natural evolution of IRS in children and adolescents. Of equal importance is the clinical imperative to have a definition of IRS both for diagnosis and interven- tion purpose. Interventions must primarily centre on lifestyle change, as they do for adults, ${ }^{1}$ the reduction of body fatness and an increase in physical fitness with a family focus. The evidence base for weight management in children and adolescents remains limited and primarily comes from university and other tertiary centres. ${ }^{29}$ Knip and Nuutinen ${ }^{30}$ describe the change in fasting insulin in a weight management programme over $5 \mathrm{y}$. Fasting insulin levels showed a significant decline over the 12 months of the intervention, but returned to baseline and higher after that time despite maintenance of relative weight lost. Given the prevalence of obesity and now IRS, interventions need to be based at a primary care and community level. Clinical studies into weight management in childhood and adolescence as well as those that consider the cardiovascular risk and IRS should be unified in their approach. Also, measures of fatness, measures of fat distribution and blood pressure, and where possible lipids, insulin and glucose should be included.

\section{KS Steinbeck ${ }^{1}$ \\ ${ }^{1}$ Metabolism \& Obesity Services, Department of Endocrinology, Royal Prince Alfred Hospital, Camperdown, Sydney, NSW,} Australia

\section{References}

1 Grundy SM, Hansen B, Smith SC, Cleeman JI, Kahn RA. Clinical management of the metabolic syndrome: report of the American heart Association/National Heart, Lung and Blood Institute. American Diabetes Association. Arterioscler. Thromb Vasc Biol 2004; 24: e19-e24.

2 Lemieux I, Almeras N, Mauriege P, Blanchet C, Dewailly E, Bergeron J, Depres JP. Prevalence of the 'hypertriglyceridemic waist' in men who participated in the Quebec Health Survey: association with atherogenic and diabetogenic metabolic risk factors. Can J Cardiol 2002; 18: 725-732.

3 Higgins PB, Gower BA, Hunter GR, Goran MI. Defining healthrelated obesity in prepubertal children. Obes res 2001; 9: 233-240.

4 Moreno LA, Pineda I, Rodriguez G, Fleta J, Sarria A, Bueno M. Waist circumference for the screening of the metabolic syndrome in children. Acta Paediatr 2002; 91: 1307-1312.

5 McCarthy HD, Ellis SM, Cole TJ. Central overweight and obesity in British Youth aged 11-16 years: cross sectional surveys of waist circumference. BMJ 2003; 326: 624.

6 Pankow JS, Jacobs DR, Steinberger J, Moran A, Sinaiko AR. Insulin resistance and cardiovascular disease risk factors in children of parents with the insulin resistance (metabolic) syndrome. Diabetes Care 2004; 27: 775-780.

7 Goran MI, Gower BA. Relation between visceral fat and disease risk in children and adolescents. Am J Clin Nutr 1999; 70: 149S$156 \mathrm{~S}$.

8 Tounian P, Aggoun Y, Dubern B, Varille V, Guy-Grand B, Sidi D, Girardet JP, Bonnet D. Presence of increased arterial stiffness of the common carotid artery and endothelial dysfunction in severely obese children: a prospective study. Lancet 2001; 358: 1400-1404.

9 Lambert M, Paradis G, O'Loughlin J, Delvin EE, Hanley JA, Levy E. Insulin resistance in a representative sample of children and adolescents from Quebec, Canada. Int J Obes Relat Metab Disord 2004; 833-841. 
10 Moran A, Jacobs DR, Steinberger J, Hong C-P, Prineas R, Luepker $R$, Sinaiko AR. Insulin resistance during puberty: results from clamp studies in 357 children. Diabetes 1999; 48: 2039-2044.

11 Bloomgarden ZT. The 1st World Congress on the insulin resistance syndrome. Diabetes Care 2004; 27: 602-609.

12 Sunehag AL, Treuth MS, Toffolo G, Butte NF, Cobelli C, Bier DM, Haymond MW. Glucose production, gluconeogenesis and insulin sensitivity in children and adolescents: an evaluation of their repeatability. Pediatr Res 2001; 50: 115-123.

13 Travers SH, Jeffers BW, Bloch CA, Hill JO, Eckel RH. Gender and Tanner stage differences in body composition and insulin sensitivity in early pubertal children. J Clin Endocrinol Metab 1995; 80: 172-178.

14 Murphy MJ, Metcalf BS, Voss LD, Jeffert AN, Kirkby J, Mallam KM, Wilkin TJ. Girls at five are more intrinsically insulin resistant than boys: the programming hypotheses revisited - the EarlyBird Study (EarlyBird 6). Pediatrics 2004; 113: 82-86.

15 Scott CR, Smith JM, Cradock MM, Pihoker C. Characteristics of youth-onset non-insulin-dependent diabetes mellitus and insulin-dependent diabetes mellitus at diagnosis. Pediatrics 1997; 100: 84-91.

16 Ehtisham S, Barrett TG, Shaw NJ. Type 2 diabetes mellitus in UK children - an emerging problem. Diabet Med 2000; 17: 867-871.

17 Chen W, Bao W, Begum S, Elkasabany A, Srinivasan SR, Berenson GS. Age related patterns of the clustering of cardiovascular risk variables of Syndrome $\mathrm{X}$ from childhood to young adulthood in a population made up of black and white subjects: the Bogalusa Heart Study. Diabetes 2000; 49: 1042-1048 .

18 Jiang X, Srinivasan SR, Webber LS, Wattigney WA, Berenson GS. Association of fasting insulin level with serum lipid and lipoprotein levels in children, adolescents and young adults. The Bogalusa heart Study. Arch Int Med 1995; 155: 190-196.

19 Srinivasan SR, Myers L, Berenson GS. Predictability of childhood adiposity and insulin for developing insulin resistance syndrome (Syndrome X) in young adulthood: the Bogalusa Heart Study. Diabetes 2002; 51: 204-209.

20 Raitakari OT, Taimela S, Porkka KV, Telama R, Valimaki I, Akerblom HK, Viikari JS. Associations between physical activity and risk factors for coronary heart disease: the Cardiovascular
Risk in Young Finns Study. Med Sci Sports Exerc 1997; 29: 10551061.

21 Despres JP, Lamarche B, Mauriege P, Cantin B, Dagenais GR, Moorjani S, Lupien PJ. Hyperinsulinemia as an independent risk factor for ischemic heart disease. N Engl J Med 1996; 3334: 952-957.

22 Goran MI, Gower BA, Treuth M, Nagy TR. Prediction of intraabdominal and subcutaneous abdominal adipose tissue in healthy pre-pubertal children. Int J Obes Relat Metab Dis 1998; 22: $549-558$.

23 Malecka-Tendera E, Molnar D. Hormonal and metabolic changes. In: Burniat W, Cole T, Lissau I, Poskitt EME (eds). Child and adolescent obesity. Causes and consequences; prevention and management. Cambridge Press: Cambridge, UK; 2002. pp 189-220.

24 Troiano RP, Flegal KM. Overweight in children and adolescents: description, epidemiology and demographics. Pediatrics 1998; 101: 497-504.

25 Magarey AM, Daniels LA, Boulton TJ. Prevalence of overweight and obesity in Australian children and adolescents: reassessment of 1985 and 1995 data against new standard international definitions. Med J Aust 2001; 174: 561-564.

26 Lillioja S, Mott DM, Spraul M, Ferraro R, Foley JE, Ravussin E. Insulin resistance and insulin secretory dysfunction as precursors of non-insulin-dependent diabetes mellitus: prospective studies in Pima Indians. N Engl J Med 1993; 329: 1988-1992.

27 McCance DR, Pettit DJ, Hanson RL, Jacobsson LTH, Bennett PH Knowler WC. Glucose, insulin concentrations and obesity in childhood and adolescence as predictors of NIDDM. Diabetologia 1994; 37: 617-623.

28 Sinha R, Fisch G, Teague B, Tamborlane WV, Banyas B, Allen K, Savoye M, Rieger V, Taksali S, Barbetta G, Sherwin RS, Caprio S. Prevalence of impaired glucose tolerance among children and adolescents with marked obesity. $N$ Engl J Med 2002; 346: 802-810.

29 Summerbell CD, Ashton V, Campbell KJ, Edmunds L, Kelly S, Waters E. Interventions for treating obesity in children (Cochrane Review). In: The Cochrane Library Issue 2. Wiley: Chichester, UK; 2004.

30 Knip M, Nuutinen O. Long-term effects of weight reduction on serum lipids and plasma insulin in obese children. Am J Clin Nutr 1993; 57: 490-493. 